Neuroepidemiology 2015;45:109-110

DOI: $10.1159 / 000435920$

\section{A Report of Stiff Person Syndrome in Tanzania with First Epidemiological Figures for Sub-Saharan Africa}

Marieke C.J. Dekker, Sarah J. Urasa, Grace Kinabo, Venance Maro, William P. Howlett

Departments of Internal Medicine and Pediatrics, Kilimanjaro Christian Medical Centre, Moshi, Tanzania

\section{Introduction}

Stiff person syndrome (SPS) is a rare autoimmune movement disorder, with prevalence estimates ranging from 0.5 to 2 per $1,000,000$ people $[1,2]$. Its diagnosis is clinical, supported by the presence of anti-GAD or anti-amphiphysin antibodies. In this paper, we describe a series of SPS patients, estimate the prevalence in Tanzania and compare it with that of high income countries.

\section{Materials and Methods}

Patients were ascertained through neurological consultation in Kilimanjaro Christian Medical Centre, a referral hospital in Northern Tanzania, during the period 2007-2014. In order to distinguish a case of SPS from tetanus, the following criteria were used: clinical criteria (table 1), time course and evidence of persistent signs at follow-up. In tetanus, symptoms will have resolved entirely. A case of SPS in this group was defined using Dalakas criteria [1]. Response to diazepam is not part of Dalakas criteria, but is commonly included as an additional diagnostic criterion [2]. Positive serology for GAD65 or amphiphysin autoantibodies; confirmation of the continuous motor unit activity by EMG. These antibody tests and EMG are still unavailable in Northern Tanzania. Patients have provided written informed consent with explicit consent for use of the photographs. The study has been approved of by the KCMC Medical Ethics Committee.

\section{Results}

A total of 9 SPS cases were reported ( 2 women and 7 men). The estimated prevalence in this patient population is 0.9 per $1,000,000$ people in the Kilimanjaro region, where our hospital is based as the main catchment area.

All patients in this study show the main clinical features of SPS (table 2). Photographs 1 and 2 of patients, in our series, illustrate the lumbar hyperlordosis and the 'railtrack sign', caused by the appearance of the hypertonic back extensor musculature (figs. 1,2).

One patient had juvenile onset of symptoms (12 years). One patient was referred abroad for advanced diagnostics and intravenous immunoglobulin therapy, with marked clinical improvement. She had a strongly positive anti-GAD antibody titer (2.1,
Table 1. Main differentiation between SPS and tetanus

\begin{tabular}{lll}
\hline Clinical feature & SPS & Tetanus \\
\hline Time course & Months or years & Weeks $^{\mathrm{a}}$ \\
Trismus & $(-)$ & ++ \\
Risus sardonicus & - & ++ \\
Laryngospasm & - & ++ \\
Abdominal rigidity & ++ & $+(+)$ \\
Lumbar hyperlordosis & +++ & + \\
Opisthotonus & $+(+)$ & +++ \\
Spasms & ++ & ++ \\
Preceding wound & $(+)$ & +++ \\
\hline
\end{tabular}

a Sometimes, months in localized forms of tetanus.

Table 2. Patient characteristics

\begin{tabular}{lcl}
\hline Epidemiological characteristics & $\mathrm{n}=9$ & Reference [5] \\
\hline Male/female distribution, \% & $7 / 2$ & $33 / 67$ \\
Age at onset, average, years & 36.7 & 41.2 \\
Age range at onset, years & $12-49$ & \\
Time elapsed before diagnosis, years & $0.8^{*}$ & \\
Clinical findings, \% & & \\
Lumbar hyperlordosis & 100 \\
Opisthotonus & 100 \\
Abdominal rigidity & 100 \\
Spasms & 100 \\
Hyperreflexia & 100 \\
Extensor plantar response & $83^{*}$ \\
Spasm-induced femoral fracture & $2 / 9$ \\
Treatment, mg & \\
Average daily dosage diazepam & $45^{* *}$ \\
Average daily dosage baclofen & 25 \\
Corticosteroid treatment & $2 / 9$ \\
\hline
\end{tabular}

Originally described anti-GAD positive SPS patient series [5]. ${ }^{*} \mathrm{n}=6$; ${ }^{* *} \mathrm{n}=7$. Where the sample is smaller than the total group number of 9 , it means that complete clinical information was not available for the remainder.

upper normal reference 1.0). Four years later, she was diagnosed with breast carcinoma. Another patient presented with stiff limb syndrome, a partial or segmental onset variant following penetrating injury, which slowly generalized over several months. One patient had a subacute onset over a course of 2 weeks with mild encephalopathic features and saccadic eye movements, but none-

\section{KARGER 125}

(c) 2015 S. Karger AG, Basel

0251-5350/15/0452-0109\$39.50/0 


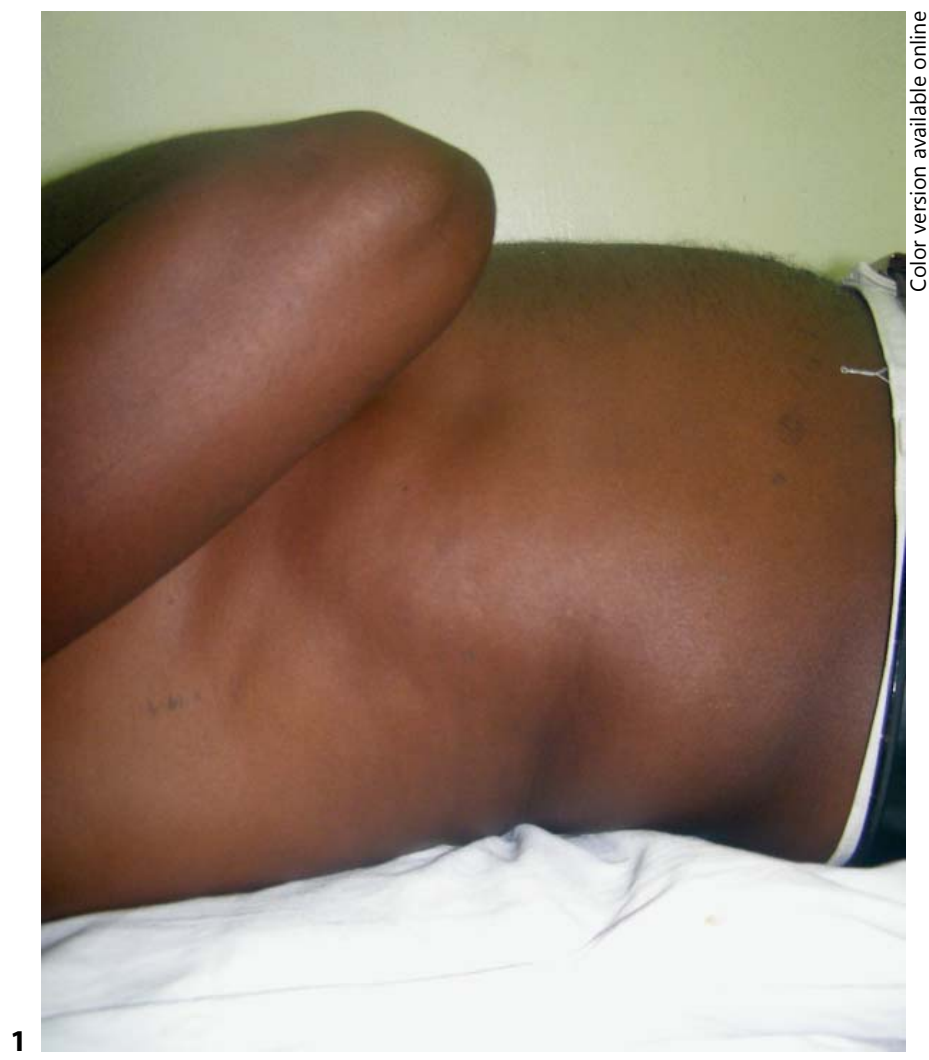

Fig. 1. Lumbar hyperlordosis.

Fig. 2. 'Railtrack sign' by marked back extensor hypertonia.

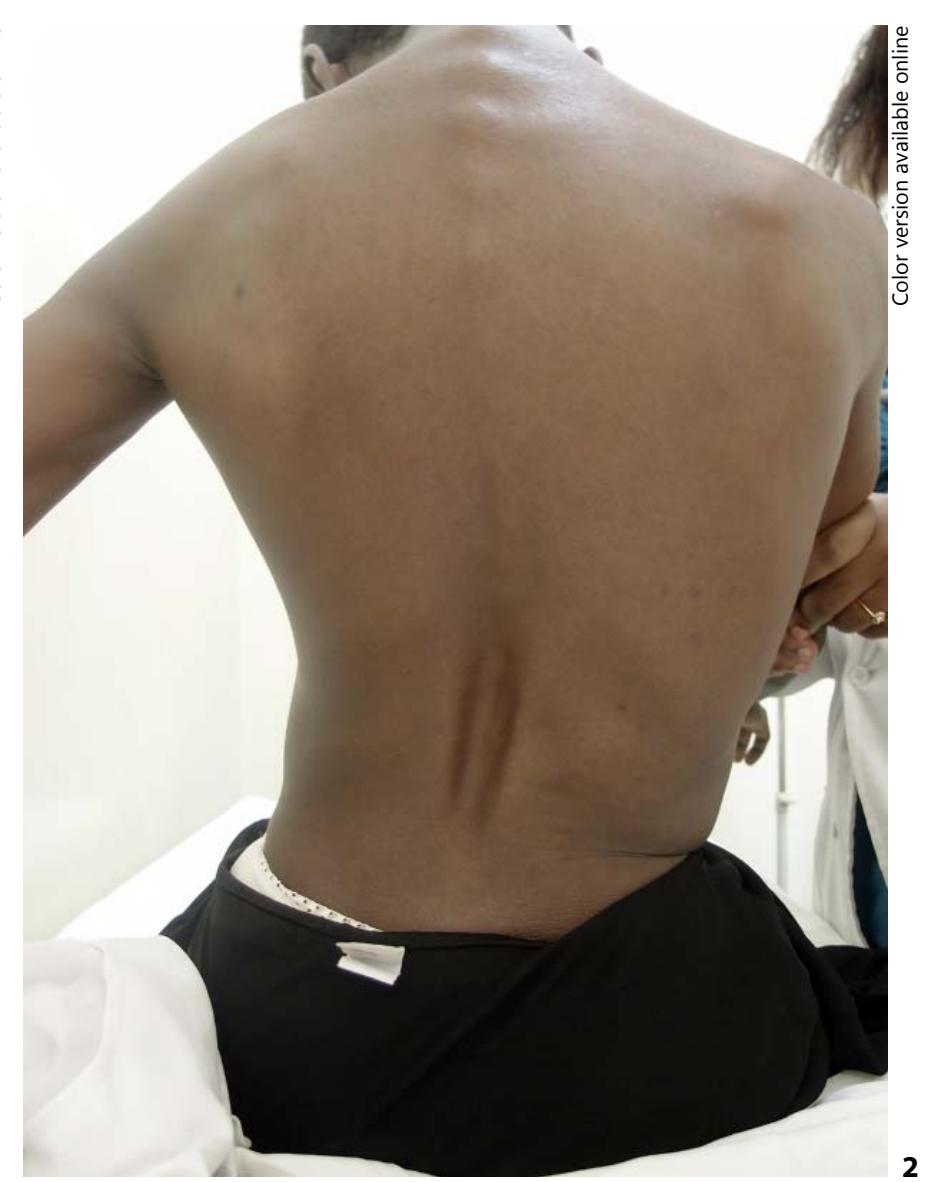

theless fulfilling the Dalakas criteria [1]. Of special interest were 2 patients who presented with a femoral fracture secondary to violent muscle spasms. All patients showed a prolonged clinical response to high-dose benzodiazepines and baclofen. The most recent patients were also treated with corticosteroids (2 of 9 patients).

\section{Discussion}

SPS occurs in Tanzania, and it is the same disorder as elsewhere. The estimated prevalence in our study compares with other populations. The numbers are small, but the male predominance may reflect the greater utilization of health-care facilities by men in this culture. Clinically, the disorder is very characteristic and can be discerned from tetanus, which is also prevalent in SubSaharan Africa. First-choice medications, such as benzodiazepines and baclofen, are available but the used dosage likely depends on the patient's financial situation. Co-treatment with a course of corticosteroids did not seem to further improve the clinical condition, but the sample size is too small to draw firm conclusions from. Our patient series shows variants described elsewhere (stiff limb syndrome, likely paraneoplastic SPS and juvenile onset SPS) $[1,3,4]$. Laboratory tests to support the clinical diagnosis of SPS [1] are unfortunately lacking in our setting.

The series reported here appears to be the first SPS prevalence report for Africa. Despite the small sample size, this compares to the range of prevalence data from other ethnic groups. There may be underestimation due to limited availability and accessibility of health-care facilities and lack of recognition of the disorder.

\section{Acknowledgments}

The initial part of the patient series described above was presented as a poster in the World Congress of Neurology in Vienna (25.09.13, P95). On behalf of all authors, the corresponding author states that there is no conflict of interest.

\section{References}

Dalakas MC: Stiff person syndrome: advances in pathogenesis and therapeutic interventions. Curr Treat Options Neurol 2009;11:102110.

Hadavi S, Noyce AJ, Leslie RD, Giovannoni G: Stiff person syndrome. Pract Neurol 2011;11:272-282.

3 Agarwal PA, Ichaporia NR: Glutamic acid decarboxylase antibody-positive paraneoplastic stiff limb syndrome associated with carcinoma of the breast. Neurol India 2010;58:449-451.

4 Clardy SL, Lennon VA, Dalmau J, Pittock SJ, Jones HR Jr, Renaud DL, Harper CM Jr, Matsumoto JY, McKeon A: Childhood onset of stiff-man syndrome. JAMA Neurol 2013;70:1531-1536.

5 Dalakas MC, Fujii M, Li M, et al: The clinical spectrum of anti-GAD antibody-positive patients with stiff-person syndrome. Neurology 2000; 55:1531-1535. 